\title{
Revisión del uso recreacional de la ketamina
}

\author{
Hidalgo Downing, E.* \\ (*) Psicólogo. Master en Drogodependencias. Miembro del equipo técnico de Energy Control (Asociación Bienestar y Desarrollo)* \\ Enviar correspondencia a: Eduardo Hidalgo Downing. C/ Los Madroños n 6, Portal 1, $3^{\circ}$ A. 28400 Villalba. Madrid. TIf: 606700699
}

\section{RESUMEN}

El clorhidrato de ketamina (ketamina), fue aprobado en 1970 para su uso médico y veterinario. Sus propiedades la convierten en un fármaco seguro y eficaz, pero también produce efectos indeseables como ensoñaciones, ilusiones y alucinaciones. Para algunos, estas desventajas médicas fueron un aliciente para su uso extrahospitalario, demostrándose pronto su enorme potencial psiquedélico e instaurándose ya en los años 70 un uso psiconáutico entre reducidos círculos de consumidores experimentados en la utilización de varias drogas.

Desde finales de los 80 y coincidiendo con la expansión de la cultura techno, las raves y las drogas de síntesis, la ketamina se introdujo en las fiestas de jóvenes que realizan un uso recreativo, consumiéndola en dosis medias o bajas, generalmente vía esnifada, y mezclándola muchas veces con otras sustancias como el éxtasis o la cocaína.

Los principales riesgos asociados al consumo son su elevado potencial de adicción psicológica, la alta tolerancia que produce y la posibilidad de dar lugar a heridas y accidentes al ser consumida en un entorno inadecuado.

Hoy en día, aún siendo una sustancia de uso minoritario, sigue expandiéndose lenta y pausadamente, por lo que sería aconsejable iniciar cuanto antes un abordaje preventivo e interventivo específico.

Palabras clave: Clorhidrato de ketamina, ketamina, uso recreacional, uso psiconáutico, potencial psiquedélico, riesgos.

\section{SUMMARY}

The use of ketamine hydrochloride (ketamine) was approved in 1970 for medical and veterinary purposes. Its properties make it a safe and effective drug, but one which also produces undesirable effects such as dreams, delusions and hallucinations. For some, these medical drawbacks were an incentive for its non-clinical use, quickly demonstrating its enormous psychedelic potential. By the seventies, a psychonautical use was already established in limited circles of consumers experienced in the use of various drugs.

Since the end of the 80s, and coinciding with the expansion of techno culture, raves and synthetic drugs, ketamine has been introduced as a recreational drug into adolescent parties where it has been consumed in low to medium doses, generally by sniffing, and frequently mixed with other substances such as ecstasy or cocaine.

The principal risks associated with its consumption are the high potential for psychological addiction, the high rate of tolerance it produces and the possibility of resulting injuries and accidents if consumed in an unsuitable environment.

Today, even though it is still a substance in minority use, it is slowly but surely gaining ground. For this reason, it would be advisable to undertake specific preventive and interventive steps to tackle the problem

Key words: Ketamine hydrochloride, ketamine, recreational use, psychonautical use, psychedelic potential, risks.

\section{INTRODUCCIÓN}

E n lo respectivo al consumo recreativo de sustancias psicoactivas, la década de los 90 fue un continuo hacer referencia a la irrupción e instauración de las denominadas drogas de síntesis. No obstante, en la práctica, fueron los derivados anfetamínicos pertenecientes al grupo de las feniletilaminas los que monopolizaron la escena del consumo recreacional. En especial el éxtasis - 3,4-metilendioximetanfetamina o MDMA (Lorenzo, 1998) - hasta el punto de que expresiones como drogas de diseño o de síntesis llegaron a convertirse en sinónimo de MDMA: referirse a ellas era, en última instancia, hacer referencia exclusiva al éxtasis. Sin embargo, en aquellos años también hicieron acto de presencia otras sustancias sintéticas, cuyo uso (minoritario y a veces incluso anecdótico) pasó casi desapercibido, quedando eclipsado por la eclosión de la 3,4 metilendioximetanfetamina.

Iniciado el siglo XXI, estas sustancias siguen estando presentes en los entornos de consumo recreacional, y algunas dan indicios de tener un cierto 
potencial para ser objeto de una mayor difusión e instauración en los próximos años.

EI CAT o metcatinona - 2-metiloamino-1 fenilpropanona (Luna \& Sánchez, 1998)- es una sustancia con una potencia de 1,5 veces la de la metanfetamina (Luna \& Sánchez, 1998). Hasta el momento, se ha extendido minoritariamente por Estados Unidos pero debido a la facilidad de su síntesis, no sería de extrañar cierta generalización de su consumo también en otros países.

El uso del GHB - gamma-hidroxibutirato (Dean \& Morgenthaler, 1998) - continúa presentando altibajos desde que, a mediados de los 90, se introdujera en los entornos de ocio nocturno bajo el reclamo de ser éxtasis líquido. Sin embargo, a falta de estudios contrastados y ateniéndose exclusivamente a indicios anecdóticos, cabría considerar que su difusión y aceptación por una pequeña porción de la población juvenil es mayor que la de hace casi una década.

El 2-CB - 4-bromo-2,5 dimetoxifenetilamina (Luna \& Sánchez, 1998) - es también una feniletilamina, en este caso con propiedades alucinógenas, en cierto sentido estaría a medio camino entre el éxtasis y la dietilamida del ácido lisérgico o LSD. Su presencia ha sido detectada ya en España, y aunque su difusión hasta el momento es mínima, son muchos los que auguran una futura expansión de su consumo recreacional (Escohotado, 1998).

Actualmente, de este grupo de sustancias, es la ketamina, sin lugar a dudas, aquella que en mayor medida y de forma más clara y patente ha afianzado su presencia y extendido su consumo. Se trata de un derivado sintético puro, es decir, hasta el momento no se conoce ningún análogo proveniente de la naturaleza (Jansen, 2001), por lo que en la farmacología de las drogodependencias pasa a ser clasificada como una droga de síntesis (Esteban, 1998). Pertenece, junto con la fenciclidina y sus derivados, al grupo de los anestésicos disociativos, y sus efectos son mixtos alucinógenos-estimulantes (Raposo, 1998). Debido a que tiene una utilidad médica y veterinaria, y a que en la mayoría de los países no está clasificada como droga de abuso, su adquisición destinada a fines extrahospitalarios no ha sido hasta ahora especialmente dificultosa, lo cual muy probablemente haya contribuido a la difusión de su consumo. Su instauración actual, al igual que sucedió con el éxtasis, ha tenido lugar especialmente en entornos asociados a las fiestas de fin de semana, la música electrónica y las raves. Su uso no deja de ser minoritario, pero es claramente una sustancia emergente con un alto potencial de difusión, hay incluso quien apunta que fácilmente podría reemplazar al LSD como una de las drogas más controvertidas de la historia, en cuanto su influencia sobre la música, las artes y la cultura en general, podría ser realmente ilimitada (Kelly, 1999).

\section{ASPECTOS FARMACOLÓGICOS}

El clorhidrato de 2-(O-clorofenil)- 2- metilamino ciclohexanona (Yate, 1996) es una sal cristalina blanca, hidrosoluble hasta el 20 \% y estable a temperatura ambiente (Collins, 1996). En la práctica clínica recibe el nombre de clorhidrato de ketamina y pertenece a la familia de las arilciclohexilaminas, de la que también forman parte la tiletamina (Cl-634) y la fenciclidina. Esta última comercializada en su día como Sernyl (Cl395) ha sido objeto de abuso entre la población juvenil, especialmente en Norteamérica donde se la conocía como polvo de ángel, peace-pill (píldora de la paz) o PCP (Collins, 1996).

El clorhidrato de ketamina (Cl-581) fue sintetizado por Calvin Stevens en 1963 con la intención de sustituir al Sernyl por un producto más seguro y médicamente más útil. Fue escogido entre doscientos derivados de la fenciclidina al constatarse que era bastante menos tóxica que esta, su acción era más rápida y breve y sus efectos psicoactivos eran menos pronunciados (Collins, 1996).

En 1965 Edward Domino la administra por primera vez a humanos y en 1970 la Food and Drug Administration (FDA) aprueba su uso médico y veterinario. Desde entonces viene usándose en el mundo entero por considerarse un anestésico seguro y eficaz. Sus indicaciones autorizadas son la inducción y el mantenimiento de la anestesia general y el control de la ansiedad ligada a procedimientos quirúrgicos y/o diagnósticos. Aunque sus efectos secundarios (que veremos más adelante), hacen que no sea el fármaco más adecuado para la inducción de anestesia en la clínica diaria, la ketamina ha demostrado ser especialmente útil en circunstancias dificultosas como el trabajo de campo o la práctica médica en períodos de guerra y en países en vías de desarrollo (Yate, 1996). Está, además, especialmente indicada en pacientes con alto riesgo de depresión de las funciones vitales (niños y ancianos), y en los que han de someterse a procedimientos repetidos: tratamiento de quemaduras y cirugía plástica y reconstructiva (Collins, 1996).

Cabe destacar, sin embargo, que el $90 \%$ de la ketamina utilizada legalmente, se destina a la clínica veterinaria, donde según la FDA estaría indicada para su uso en gatos y primates, aunque en la práctica se utiliza con muchos otros animales, a excepción de los destinados al consumo humano, en cuanto en este caso su utilización está expresamente prohibida (Booth, 1988).

La patente pertenece a Parke Davis, que la comercializa en España con el nombre de Ketolar como anestésico de uso hospitalario. En veterinaria lo distribuye Rhône Merieux con la denominación de Imalgéne. 
Técnicamente la ketamina es definida como un anestésico disociativo, término que acuñó Edward Domino (1965) tras administrarla por primera vez a humanos. A diferencia de los fármacos tradicionales (barbitúricos o narcóticos), la anestesia que produce la ketamina viene determinada por la disrupción funcional (disociación) del Sistema Nervioso Central (Collins, 1996): según indica el EEG, se produce una depresión del sistema talomocortical (supresión o alteración de la información auditiva y somatosensorial) junto a una activación del sistema límbico (estimulación de la corteza visual), lo cual da lugar a un peculiar estado de inconsciencia en el que la persona no está dormida sino desconectada de su cuerpo y de su entorno. Bajo los efectos de la ketamina el cerebro sería incapaz de interpretar la información sensorial procedente de los sentidos, por lo que se podría decir que queda aislado, desconectado o separado del cuerpo (Collins, 1996). En el plano neuroquímico, la acción de la ketamina parece estar determinada, a su vez, por el bloqueo de los canales de los receptores N-metil-D-aspartato (NMDA), implicados en la coordinación e integración de las funciones corporales y la mente consciente (Kelly, 1999). La ketamina, mediante el bloqueo de los receptores NMDA, impediría la conexión de las neuronas con el neurotransmisor glutamato, que cumple una función fundamental en las áreas cerebrales que regulan la memoria, la emoción, el lenguaje, la personalidad y la capacidad de aprender de la experiencia (Jansen, 1997; 2001). De este modo, los efectos que volvemos a obtener son la descoordinación mentecuerpo, la activación de unas áreas cerebrales y la inhibición de otras y la drástica reducción de la información sensorial.

Existen además otras características de la ketamina que hacen de ella un fármaco peculiar:

- Produce una mínima depresión respiratoria, referida más al volumen de aire inspirado que a la frecuencia de las inspiraciones. Generalmente, esta depresión dura únicamente de uno a tres minutos y luego se restablece la respiración normal. No obstante, existe el riesgo de una depresión respiratoria grave e impredecible, sobre todo con dosis altas o con una administración demasiado rápida (Collins, 1996).

- Produce una ligera estimulación del aparato cardiovascular, aumentando la frecuencia y el gasto cardiaco así como la presión arterial, lo cual puede ser perjudicial para personas con antecedentes de accidentes cerebrovasculares, hipertensión o descompensación cardiaca (Collins, 1996).

- Conserva el tono de los músculos esqueléticos e incluso lo puede aumentar (Collins, 1996).

- Los reflejos laríngeos y faríngeos permanecen activos, conservando el tono de los músculos mandibulares y de la lengua, por lo que se puede ingerir saliva, moco o material regurgitado, disminuyendo notablemente el riesgo de ahogo accidental (Collins, 1996).

- Produce analgesia profunda al dolor somático (Collins, 1996).

Sin embargo, al igual que ocurre con otros anestésicos generales, el clorhidrato de ketamina presenta el inconveniente de producir reacciones psicológicas indeseables en un 20-30 \% de los pacientes (Collins, 1996). Este tipo de reacciones se dan en el estado de emergencia o emergente, es decir, en el momento del despertar, cuando el paciente comienza a salir del estado de anestesia, e incluyen fenómenos tales como delirio, ensoñaciones, desorientación, desrealización, despersonalización, cambios en el estado de ánimo y afecto, ilusiones y alucinaciones (Collins, 1996). En la clínica, en cuanto estas reacciones se consideran médicamente indeseables, se trata de reducir su ocurrencia administrando fármacos como las benzodiacepinas o los barbitúricos (Collins, 1996). Sin embargo, lo que para los médicos es una desventaja de la ketamina, para las personas interesadas en experimentar estados alterados de conciencia constituye toda una virtud, por lo que tratan de obtener estas alucinaciones y delirios sin entrar antes en el estado de anestesia. Esto lo consiguen administrándose dosis que generalmente no sobrepasan el 10-25 $\%$ de las utilizadas en el ámbito clínico, de esta manera alcanzarían concentraciones plasmáticas de ketamina similares a las que se dan al producirse los fenómenos de emergencia tras la anestesia general hospitalaria (Hansen et al, 1988).

Lo cierto es que este uso recreacional en busca de las alucinaciones e ilusiones que experimentaban los pacientes clínicos, llevó a descubrir que la ketamina posee unas potentísimas propiedades psiquedélicas (mayores incluso que las del LSD) y a ser incluida junto a la escopolamina, hiosciamina y atropina en el reducido grupo de sustancias que realmente pueden recibir el calificativo literal de alucinógenas (Fericgla, 2000).

\section{DIFUSIÓN DEL CONSUMO}

El uso recreacional del clorhidrato de ketamina comenzó y se extendió de forma paralela a su implantación como anestésico a partir de 1970, en cuanto numerosos psiconautas (exploradores de la conciencia) que en los años 60 habían utilizado LSD y mescalina continuaron sus experimentaciones en décadas posteriores con las nuevas sustancias que se ponían a su disposición, entre ellas la ketamina (Rollo \& Samorini, 1999). 
Parece ser que hasta mediados de los 80, el uso recreacional se difundió principalmente entre consumidores adultos, adaptados, con buenas condiciones socioeconómicas y académica y profesionalmente bien cualificados (Weil \& Rosen, 1999; Jansen, 2001). Muchos eran médicos y personal hospitalario, y también abundaban los psicólogos y los intelectuales de diverso tipo. En definitiva, solían ser personas preparadas e integradas socialmente que con esta droga trataban de experimentar estados alterados de conciencia en busca de un crecimiento interior (Moore \& Alltounian, 1978; Lilly, 1997). Con el tiempo, sin embargo, el consumo se fue extendiendo y abarcó a otro tipo de consumidores con motivaciones y características sociodemográficas diferentes: jóvenes y adolescentes, principalmente de la cultura techno, que realizaban un uso lúdico de la sustancia, muchas veces mezclándola con otras drogas. Actualmente coexisten estos dos grupos de consumidores, pero esta vez es mucho más numeroso el segundo colectivo y su correspondiente modo de utilizar la ketamina.

La inflexión que supuso la popularización del uso recreativo de esta sustancia parece producirse en la segunda mitad de la década de los 80 , coincidiendo con la eclosión de la cultura techno. Durante este período, parece ser que las fiestas en las playas de Goa (India) habrían jugado un papel similar al que pudo haber tenido Ibiza en la popularización del éxtasis (Jansen, 2001). En India, al igual que en otros países donde los recursos escasean, la ketamina puede ser obtenida fácilmente a lo largo y ancho de todo el país, por tratarse de un anestésico muy barato. Todo indicaría que la ketamina sufrió una popularización inicial en las mencionadas fiestas de Goa, al ser difundido su uso principalmente por turistas norteamericanos, que ya conocían la sustancia y que en India no encontraron ningún tipo de impedimento para adquirirla libremente. De Goa regresaría la onda expansiva a Estados Unidos, adquiriendo gran popularidad en determinados locales nocturnos de Nueva York, Miami y otras ciudades norteamericanas. Con el tiempo el consumo se fue extendiendo y afianzando hasta el punto de que actualmente parece ser la sustancia de moda en Estados Unidos y se habla incluso de una generación-k de jóvenes cuya droga de abuso preferida sería la ketamina (Gayo, 1999). Su consumo ha sido detectado en lugares tan dispares como Tailandia, Sudáfrica, Australia o Italia (Jansen, 2001).

La entrada en Europa se produjo a principios de los años 90, principalmente vía Reino Unido y en forma de comprimidos vendidos como éxtasis. Esto hizo que por aquel entonces la ketamina adquiriese muy mala reputación: Ios consumidores, que esperaban sentir los efectos del éxtasis, se encontraban con una sustancia con efectos totalmente diferentes y para los cuales no estaban preparados (Dalgarno, 1996; Jansen, 2001). Al poco tiempo se deshizo el entuerto y los usuarios comenzaron a adquirir directamente ketamina y a administrásela de forma esnifada. Desde entonces, su presencia se ha ido afianzando, y hoy en día, aún sin ser masivo, cuenta con una notable difusión, especialmente en el Reino Unido y en los Países del Este (Kelly, 1999).

En España los primeros decomisos policiales datan de mediados de los años 90, aunque su presencia en las salas de fiesta parece remontarse a principios de esa misma década. Gamella y Roldán (1997) comentan haber localizado algunos usuarios experimentales de ketamina en su estudio de campo sobre patrones de consumo de drogas de síntesis en España. Por su parte, el grupo Energy Control (Asociación Bienestar y Desarrollo), que trabaja en el área de reducción de riesgos asociados al consumo de drogas, ha entrado en contacto directo con numerosas personas y colectivos que consumen o han consumido la sustancia y tiene noticia de su uso cada vez más extendido por toda la geografía española. De hecho, en las evaluaciones que este mismo grupo realizó durante el año 2000 en los espacios de ocio juvenil de Madrid y Barcelona, fue pasado un cuestionario a una muestra de personas escogidas de forma aleatoria de entre todas aquellas que se acercaban al stand. Entre otras cuestiones se preguntaba por las sustancias consumidas alguna vez en la vida, obteniéndose como resultado que en Madrid, de una muestra total de 112 personas, $4(3,57 \%)$ decía haber consumido alguna vez ketamina con fines recreativos. En Barcelona de una muestra de 291 personas, 61 (21 \%) declaraban haberla consumido alguna vez, y 10 personas $(3,4 \%)$ se consideraban consumidores habituales. Evidentemente, el valor de estos datos no es más que anecdótico, debido al reducido tamaño de la muestra y a la metodología empleada, no obstante pone de manifiesto una vez más que la droga está presente en el ámbito del ocio nocturno, y anima a continuar realizando investigaciones al respecto. Respecto a la diferencia en los porcentajes obtenidos en Madrid y Barcelona, cabe considerar que se deben principalmente a que en la primera ciudad las intervenciones se realizaron en discotecas y macrodiscotecas techno con un público bastante joven, mientras que en la segunda, gran parte de las actuaciones se realizaron en fiestas rave donde el consumo en general es más elevado y donde precisamente más se ha instaurado y extendido el uso recreacional de la ketamina.

Más allá de la constatación directa del consumo de esta sustancia, existen varios indicios que parecen señalar un uso emergente del clorhidrato de ketamina, principalmente entre la población juvenil. En España, la Junta de Gobierno del Colegio Oficial de Farmacéuticos se vio obligada en noviembre de 1999 a pasar una circular a las oficinas de Farmacia recordando que no podrá dispensarse clorhidrato de ketamina excepto a los hospitales. Pocos días antes, un 
periodista de Diario 16 (1999) había redactado un artículo en el que denunciaba la falta de control en la venta de esta sustancia: él mismo la había podido comprar sin receta y sin ningún tipo de acreditación. Ese mismo año, la Organización de Consumidores y Usuarios (OCU), presentó un informe que destapaba presuntas irregularidades en la dispensación de fármacos de uso veterinario. Esta organización pudo adquirir clorhidrato de ketamina en 20 provincias españolas: en el $90 \%$ de los casos fue dispensada sin receta y sin que el cliente tuviera que mostrar credencial alguna. El caso ha llegado hasta la Audiencia Nacional, ya que se sospecha que el mercado de la droga pueda estar abasteciéndose de partidas de ketamina adquiridas sin control en establecimientos dispensadores de especialidades farmacéuticas de uso veterinario (Diario 16 1999; 2000).

En Estado Unidos, la Drug Enforcement Agency (DEA) incluyó la ketamina en la Lista III de las sustancias controladas en Agosto de 1999, tras haber aumentado considerablemente las emergencias hospitalarias asociadas a su abuso y las incautaciones policiales de partidas destinadas al tráfico ilícito.

Por otra parte, la ketamina parece contar en los últimos años con una presencia creciente en los medios de expresión de la cultura popular, que muchas veces puede aportar pistas importantes sobre lo que se lleva o no en la subcultura de la droga. Se le dedican artículos y reportajes en los medios de prensa escrita: en España El País (1998, 2001), El Mundo (1998), Diario 16 (1999; 2000), Interviú (Gallo, 1999), Ulises: Revista de viajes interiores (Rollo \& Samorini, 1999; Hidalgo, 2001), Cáñamo (Samper, 2001; Gallego 2001), en el extranjero revistas como High Times (Sputz, 1989) o Time Magazine (Cloud, 1997). Aparece mencionada en películas cinematográficas: Armagedón (1998) o en series televisivas: (Expediente $X$, 1997). Los grupos de la escena techno hablan de ella en sus canciones: Chemical Brothers, Mrs. Wood, The Higher Intelligence Agency. Los novelistas la mencionan o la convierten en el núcleo central de sus obras (muchas veces autobiográficas): Journeys Into The Bright World (Moore \& Altounian, 1978), The Scientist (Lilly, 1997), Design for Dying (Leary, 1997), The Ecstasy Club (Rushkoff, 1997), Disco Bloodbath (James, 1999). Aparecen auténticos manuales de consumo realizados por y para los miembros de la "comunidad psiquedélica": The Essential Psyquedelic Guide (Turner, 1994), The Little Book of Ketamine (Kelly, 1999). Se venden camisetas en puestos callejeros y por correo con inscripciones como "Ketamine 25 grs. of pure teckno smack" o "Special K, 100 \% psicoactive". Por último, existen en la World Wide Web numerosos documentos que abordan la ketamina desde diversas perspectivas, algunos están realizados por los propios usuarios o miembros de la comunidad psiquedélica (www.lycaeum.org), otros pertenecen a asociaciones y ONGs (www.energycontrol.org) y algunos han sido escritos por y para los profesionales del área de las drogodependencias (Hidalgo, 2000), en la mayoría de los casos la información que en ellos se ofrece está encaminada a informar sobre los riesgos asociados al consumo con el fin de reducir su ocurrencia.

\section{CUESTIONES BASICAS SOBRE LA SUSTANCIA Y SU USO RECREATIVO}

En vista del creciente interés que el consumo de ketamina está despertando en la cultura popular, parece conveniente que los especialistas en adicciones adquiramos cuanto antes unos conocimientos básicos, pues aunque todavía es pronto para hacer predicciones, no sería de extrañar que en un plazo de tiempo no excesivamente largo, la difusión en España adquiriese las dimensiones que ha tomado en otros lugares como Rusia o Estados Unidos, donde el consumo emergente de ketamina comienza a inquietar ya a la sanidad pública (DEA, 1999). A continuación, por lo tanto, se tratará de exponer brevemente las características principales de esta sustancia y de su uso recreacional.

\section{Presentación}

El clorhidrato de ketamina es una sal cristalina blanca que en su presentación farmacéutica aparece como un líquido inyectable, incoloro, transparente y estable a temperatura ambiente (Collins, 1996). En el mercado ilícito, además de su presentación líquida, puede presentarse en forma de polvo o cristales blancos, comprimidos y cápsulas.

\section{Composición}

Los preparados farmacéuticos en solución inyectable están compuestos de clorhidrato de ketamina más conservantes. Generalmente la cantidad del conservante es de 0,1 mg, y según los casos puede tratarse de cloruro de bencetonio, clorobutanol, cloruro de sodio o conservante E-219 (Jansen, 2001). Dada la ínfima cantidad en que estos se presentan, no son de esperar diferencias destacables entre unos y otros preparados, así como serían poco probables los efectos adversos asociados al consumo de estos conservantes. No obstante, esto habría de ser objeto de estudios en profundidad, pues el clorobutanol ha demostrado ser neurotóxico en estudios con animales (Malinovsky, 1993) y el cloruro de bencetonio a grandes dosis podría provocar delirios (Jansen 2001), por lo que tal vez el uso continuado y abusivo de ketamina con estos aditivos no esté exento de riesgos.

En el mercado ilícito, la composición de la ketamina puede ser muy variada, en cuanto como sucede 
con otras drogas, es habitual añadir todo tipo de adulterantes para aumentar el margen de beneficio económico. Hasta el momento, se cuenta con análisis toxicológicos de muestras de comprimidos vendidos como éxtasis que en realidad contenían una mezcla de ketamina y anfetamina, efedrina, selegilina, cafeína o manitol (www.ecstasy.org).

\section{Definición}

Entre los consumidores la ketamina generalmente es definida como un anestésico para caballos y elefantes. Esta última variedad, que vendría de la India y sería mucho más potente, pasa por ser la más preciada. Lo cierto es que como ya hemos comentado anteriormente, la ketamina es un anestésico disociativo usado tanto en medicina como en veterinaria, por otra parte, aunque es cierto que en algunos países existe un contrabando ilegal de ketamina procedente de la India (Kelly, 1999; Jansen, 2001), no existe una variedad más potente que sirva para anestesiar elefantes, y en los contados y excepcionales casos en que se ha usado para este menester (normalmente se usan opiáceos), lo que se utiliza es una dosis mayor que la usada para anestesiar animales más pequeños.

\section{Denominación}

Para referirse a la ketamina los consumidores generalmente utilizan alguno de los siguientes términos: K, Ket, Keta, Ketamina, Special-K, Kit-Kat, Vitamina-K, Super-K, Super-Ácido, etc (Kelly, 1999). Curiosamente los británicos también la denominan Horse (caballo) y Cat-Valium (valium para gatos), precisamente por tratarse de un anestésico utilizado en estos animales. La combinaciones de ketamina con otras drogas también reciben nombres específicos entre sus consumidores: CK o Calvin Klain (ketamina y cocaína), CK-One (cocaína, ketamina y éxtasis), E-K (éxtasis y ketamina), Mary-Kay (cannabis y ketamina). Para referirse al peculiar estado que la sustancia provoca, los consumidores hablan del Estado K, dicen que han visitado K-land o la Zona-K. El término anglosajón K-Hole (Agujero-K), designa el estado casi de inconsciencia que se alcanza con dosis altas de ketamina, que producen una experiencia psiquedélica muy intensa al tiempo que dejan el cuerpo prácticamente anulado (Jansen, 2001). En Gran Bretaña y Estados Unidos (en determinados ámbitos incluso en España), los usuarios de ketamina reciben el nombre de $K$ Heads (Cabezas-K), K-Holers, Ketters o Ketapowers.

\section{Estatus legal}

La ketamina no está incluida en los listados internacionales de sustancias psicotrópicas o estupefacientes, es decir, no está clasificada o definida como droga. En España, esta sustancia es considerada legalmente un medicamento y, por lo tanto, en princi- pio no le serían aplicables la Ley de Protección de la Seguridad Ciudadana ni el Código Penal. Aún así, al ser un medicamento, está sujeta a la Ley 25/90 (Ley del Medicamento).

Fuera de nuestro país, el 12 de agosto de 1999, la DEA incluyó la ketamina en la Lista III de las sustancias controladas en Estados Unidos, al haber aumentado los decomisos policiales y las urgencias médicas asociadas al uso/abuso de ketamina (DEA 1999). Por ello, aunque continúa teniendo un uso médico y veterinario, se ha aumentado el control sobre su producción, distribución y venta, y se castiga con mayor dureza su posesión, producción, distribución y venta ilícitas (IPRC, 1997), en Nueva York, por ejemplo, la posesión ilícita se penaliza hasta con siete años de prisión.

También en Estados Unidos, la ketamina está incluida en el Acta para la Prevención y el Castigo de la Violación Sexual Inducida por Drogas (que en algunos estados funciona como una ley), por considerarse que tiene el mismo potencial para facilitar la violación que el GHB o el Rohipnol (Barayuga, 1997).

\section{Métodos de producción}

La ketamina, a diferencia del éxtasis y de otras sustancias, es una droga difícil de sintetizar. Hasta el día de hoy no ha sido localizado ningún laboratorio clandestino que la sintetizase (DEA, 1999). La mayor parte de la ketamina consumida clandestinamente es obtenida mediante engaños al personal que la dispensa o mediante robos en las farmacias y clínicas veterinarias (DEA, 1999). También se importa de contrabando de países donde el control de su venta es mucho menor (por ejemplo: India, Méjico y Países del Este) o se compra vía Internet a distribuidores que exigen poca o ninguna acreditación para adquirirla (Kelly 1999; Jansen, 2001).

Los preparados farmacéuticos de ketamina que se presentan en forma líquida, son posteriormente convertidos en polvo mediante el simple método de cocerlos al baño maría, calentarlos en el microondas o meterlos en el horno a 90-95 $\mathrm{C}_{\infty}$, (hay incluso quien los calienta en una cuchara encima del fuego de la cocina o simplemente con un mechero), hasta que se evapora el líquido. Queda entonces un polvo blanco en forma de granos grandes de sal. Este polvo es raspado con una cuchilla para desprenderlo del recipiente y machacado para hacerlo más fino y apto para ser esnifado.

El polvo obtenido normalmente es vendido tal cual, aunque en algunos casos ha sido prensado en forma de comprimidos para venderlos como si fueran pastillas de éxtasis.

Los preparados farmacéuticos de ketamina tienen generalmente un precio inferior a las mil pesetas, (por ejemplo: Ketolar 50 mg/ml, un vial de $10 \mathrm{ml}$. -medio 
gramo de clorhidrato de ketamina en polvo- de Parke Davis S.L. cuesta 813 pesetas). En el mercado ilícito, dependiendo de varios factores (vendedor, comprador, lugar, momento...), puede alcanzar precios cinco o diez veces superiores.

Aunque muchos consumidores o pequeños traficantes logran obtener la ketamina por su propia cuenta (aprovechando contactos en veterinaria o anestesiología, o agudizando el ingenio), lo cierto es que en el extranjero existen ya pequeños y medianos grupos dedicados al contrabando y distribución ilegal de la sustancia a gran escala (Media Awareness Project, 1999).

En España, el modo habitual de conseguir esta droga continúa siendo el engaño a las personas facultadas para dispensarlo (EL PAÍS, 2001) o la utilización de contactos introducidos en la práctica médica y veterinaria. También es relativamente frecuente la importación ilegal de pequeñas cantidades procedentes de otros países, principalmente del Reino Unido.

\section{Métodos de consumo}

La ketamina presenta una enorme versatilidad en cuanto a vías de administración se refiere, pudiendo ser consumida vía oral, intranasal, rectal, pulmonar, intravenosa e intramuscular. En el ámbito recreativo las vías más habitualmente utilizadas son la oral y la intranasal (esnifada) y en el ámbito psiconáutico principalmente la intramuscular, puesto que comparativamente es la que produce los efectos más intensos y duraderos.

Sin embargo, cabe puntualizar que el uso intramuscular también se ha introducido de forma minoritaria en determinados círculos, principalmente de asiduos a las fiestas rave, como así lo ha constatado el grupo Energy Control, a quienes en su stand de reducción de riesgos han llegado a serles solicitadas jeringuillas para la administración de ketamina.

\section{Aparición y duración de los efectos}

La aparición y duración de los efectos depende, evidentemente, de la dosis y de la vía de adminisrtración utilizada. En el caso de la vía oral, los efectos aparecen entre los 5 y los 20 minutos y se pueden prolongar unas dos horas, retornándose a la línea base a las 4-8 horas (Kelly, 1999). Con la vía intranasal los efectos aparecen a los 5-15 minutos y pueden durar otros 30 minutos más, retornándose a la línea base aproximadamente a las dos horas (Kelly, 1999). Por último, la vía intramuscular antes de los 5 minutos produce efectos que se pueden prolongar hasta una hora y media, retornando a la línea base a las 4-7 horas (Kelly, 1999).

\section{Dosificación}

La dosificación variará también según la vía de administración. Para la vía intramuscular, el umbral se sitúa en los 10-15 mg, obteniéndose ligeros efectos con dosis de 15 a $30 \mathrm{mg}$. Efectos suaves con dosis de 25 a 50 mg, y fuertes con 40-100 mg. Los efectos psiquedélicos plenos se obtendrían con cantidades de 60 a 125 mg, (Kelly, 1999).

El umbral de la vía oral se sitúa en los $40-50$ mg, obteniéndose ligeros efectos psiquedélicos con dosis de 50-100 mg, suaves con 75-300 mg, y fuertes con 200-450 mg (Kelly, 1999).

La administración intranasal (esnifada), tiene su umbral en los 10-15 mg, y se obtienen efectos ligeros con $15-30 \mathrm{mg}$, suaves con $30-75 \mathrm{mg}$, y fuertes con 60-125 mg. Las experiencias psiquedélicas plenas se alcanzarían por esta vía con dosis de 100 a 250 mg, (Kelly, 1999).

\section{Efectos}

Para hacerse una primera idea de los efectos de la ketamina, puede ser interesante recurrir a las investigaciones que el neurocientífico J. C. Lilly realizó autoadministrándose distintas cantidades de esta sustancia. En su obra autobiográfica The scientist (Lilly, 1997), el autor relata como durante unos pocos minutos tras el consumo (el tiempo exacto depende de la vía de administración) no se siente ningún tipo de efecto. Después estos aparecen de modo súbito y progresan muy rápidamente hasta que se llega a una estabilización, a una meseta, que puede durar de 10 a 45 minutos según como se consuma la sustancia. Las experiencias que se viven en esta meseta dependen a su vez de la cantidad de ketamina consumida. Después, según decrecen los niveles de ketamina en plasma, el consumidor puede analizar y hasta cierto punto controlar los efectos y fenómenos que ocurren durante un período de 20 a 60 minutos o más (según la dosis y la vía de administración), esto es lo que se conoce como el viaje suave (soft-trip).

Como ya dijimos, Lilly probó en sí mismo diferentes dosis de ketamina, determinando qué efectos se correspondían con cada una de ellas. El autor, que utilizaba la vía intramuscular, estableció que con 10 mg, los efectos eran prácticamente indetectables. Con 20 mg, se producía un cosquilleo en la piel y un ligero aumento de la energía corporal, aunque no había cambios en el campo visual o en la percepción de sí mismo. 30 mg, producían cambios perceptivos, y con los ojos cerrados aparecían imágenes muy vivas. Con 75 mg, Lilly llegaba a sentir que abandonaba su cuerpo y participaba en las escenas que antes eran sólo imágenes visuales, llegando a comunicarse e interactuar con seres extraños que habitaban esos mundos ketamínicos. Administrándose 150 mg, el autor perdía su sentido de identidad: "Yo como individuo desapare- 
cía" (Lilly 1997, p 173) y con 300 mg, era incapaz de describir lo que sucedía, le parecía como si hubiese entrado en el vacío, por lo que consideraba esta dosis demasiado elevada para la exploración psiquedélica, siendo de la opinión de que las "regiones" y experiencias alcanzables con estas cantidades de ketamina "estaban más allá del entendimiento humano" (Lilly, 1997).

En base a los escritos de Lilly, los relatos de numerosos consumidores y los estudios de investigadores como K. L. R. Jansen (2001), podemos afirmar que la ketamina, consumida en pequeñas dosis produce unos efectos similares a los de una borrachera por alcohol: pérdida de coordinación, dificultad para andar, hablar y moverse, etc. Además, al igual que sucede con el alcohol, que es un depresor del sistema nervioso central pero que muchas veces puede dar la impresión de estimular más que deprimir al consumidor, la ketamina, aún siendo un anestésico, cuando se consume en dosis medias y bajas puede actuar como un estimulante, permitiendo al usuario realizar actividades como bailar e interactuar aceptablemente con el entorno. De hecho, con tales dosis, la ketamina acelera la tasa cardiaca y no deprime la respiración (Tweed et al, 1972). Si además se mezcla, como a menudo sucede, con drogas como los derivados anfetamínicos o la cocaína, que contrarrestan la disociación y la descoordinación corporal, los efectos del special-k podrían ser vividos por los usuarios como activadores más que como inhibidores.

La forma de consumo más extendida actualmente parece ser precisamente esta: los consumidores utilizan pequeñas o medias cantidades de special-k como complemento a su "menú psicotrópico" principal, compuesto fundamentalmente de éxtasis y cocaína. La intención básicamente es la de experimentar ligeramente los efectos disociativos de la ketamina, incrementar la sensación de estar colocado y dar un pequeño toque psiquedélico (alcanzable con dosis medias) al "banquete químico" del fin de semana. Muchos usuarios la utilizan también al final de la fiesta para contrarrestar los efectos desagradables de drogas como los derivados anfetamínicos y la cocaína (tensión mandibular, agobio, cansancio, etc.) ya que el efecto anestésico y analgésico de la ketamina elimina toda esta gama de sensaciones corporales y/o psicológicas.

Tal vez, en este punto sea conveniente hacer un pequeño inciso para tratar de dar una explicación a como una sustancia en principio más indicada para la experimentación trascendental y psiconáutica en contextos tranquilos (debido a sus propiedades anestésicas y altamente psiquedélicas), consigue afianzar y extender su presencia en el ambiente del consumo recreativo. Recapitulando, podemos considerar que los usuarios en los espacios de ocio nocturno generalmente toman dosis bajas con las que el embotamien- to corporal y la disociación no son tan marcadas como para impedir la interacción con el entorno. Ya hemos señalado también, como estas dosis producen una aceleración de la tasa cardiaca, más aún cuando se mezclan con sustancias como la cocaína, el éxtasis o la metanfetamina, que también contrarrestan el embotamiento y la disociación. La ketamina, por su parte, no sólo reduce los efectos secundarios derivados del consumo de estimulantes, sino que también aporta sus propios efectos: intensa y rápida embriaguez, ligeros efectos disociativos (sensación de flotar), moderadas alteraciones perceptivas (visión y audición distorsionadas, percepciones extrañas del propio cuerpo), alucinaciones (visiones con los ojos cerrados) y cierta euforia. A fin de cuentas, la ketamina altera el estado ordinario de conciencia y esto para muchas personas es gratificante. Los efectos directos del special-k, más los que suma y resta de su interacción con otras sustancias, para algunas personas son agradables, divertidos o curiosos. Si además, como ya hemos visto en apartados anteriores, se trata de una sustancia legal, que a veces se puede conseguir a bajo precio, con control sanitario, sin adulterantes y en dosis perfectamente medidas, es comprensible que muchos usuarios de drogas se sientan atraídos por probarla: en un mercado ilícito plagado de adulteraciones, algo así no se encuentra habitualmente.

Por otra parte, es evidente que las dosis altas son incompatibles con la interacción social, el baile y los entornos de marcha, y es por ello que el consumidor recreativo, que busca alterar su estado ordinario de conciencia en contextos sociales asociados a la diversión, se infradosifica: toma únicamente dosis pequeñitas que no le aportan los efectos plenos de la ketamina. El riesgo está en que a diferencia del consumidor psiconáutico, (que utiliza dosis altas en una única toma), el consumidor recreativo tiende a adoptar el patrón de consumo propio de sustancias como la cocaína: pequeñas dosis cada poco tiempo. De esta forma no llega a alcanzar estados anestésicos, disociativos o realmente psiquedélicos, pero favorece el desarrollo de tolerancia y adicción, y siguiendo una pauta de infradosificación repetida, llega a sobredosificarse, consumiendo, en ocasiones, dosis alarmantemente altas (incluso varios gramos en una sola noche), lo cual incrementa enormemente los riesgos físicos, psíquicos y contextuales.

Hechas estas aclaraciones, continuemos con el relato de los efectos de la ketamina: Estos suelen comenzar con un zumbido en los oídos, que va incrementando durante unos dos minutos. La tasa cardiaca aumenta, produciéndose una ligera taquicardia, al mismo tiempo que se pueden dar tenues temblores de piernas y una moderada y pasajera depresión respiratoria que el consumidor puede vivir como si se quedara levemente falto de aire. Estos efectos suelen ser considerados como criterios indicativos para esta- 
blecer si la ketamina es o no de buena calidad y para identificar el momento de la subida (Rollo \& Samorini, 1999).

Posteriormente, dependiendo de la dosis, se pierde en mayor o menor medida el control corporal, la coordinación y el equilibrio. El cuerpo se va quedando entumecido y la audición y la visión se distorsionan. Se pierden algunas frecuencias de sonido (Plourde et al, 1997) y, o bien no se oye prácticamente nada (a dosis altas) o todo suena mal y más alto de lo normal. Con los ojos cerrados o con luz tenue es frecuente que se vean imágenes, y cuando se mantienen abiertos la visión puede ser borrosa o doble y las luces brillantes pueden molestar. También son frecuentes las alteraciones en la percepción del cuerpo, que a veces dan lugar a que el usuario se vea a sí mismo grotescamente deformado o sienta como si su cuerpo estuviera compuesto de materiales extraños (plástico, madera, metal) y funcionase por medio de poleas y otros elementos mecánicos (Dalgarno, 1996).

Si la dosis administrada ha sido alta, todos estos efectos se ven multiplicados, y la experiencia toma un carácter netamente psiquedélico. Los sentidos (olfato, gusto, tacto, visión, audición) quedan anulados o al menos notablemente alterados (Kelly, 1999). Desaparecen las sensaciones de dolor, y la noción del tiempo se desvanece, confluyendo en un mismo momento presente, pasado y futuro. Cuesta mucho hablar, aunque a veces pueden repetirse persistentemente palabras que en ocasiones carecen de significado (Jansen, 2001). Estar de pie, andar o moverse resulta casi imposible y, en caso de que se logre hacerlo, requiere una determinación y concentración sobrehumanas, y se realiza de manera extremadamente torpe, lenta y dificultosa (Jansen, 2001). En el plano emocional, lo más común es que el usuario se sienta en un "peculiar estado de alta indiferencia" viéndose a sí mismo como "un ser altamente neutral" (Lilly 1997, p 171172). Sólo después de que la experiencia psicodélica comience a remitir, el consumidor irá retomando sus emociones. Es entonces cuando puede que vea las cosas desde una óptica benévola, optimista y jovial, o bien se asuste o se preocupe y enfoque las situaciones desde la paranoia y la negatividad. En cuanto a la sensualidad y la sexualidad, ambas se ven completamente anuladas durante la experiencia psiquedélica. Una vez más, cuando esta vaya remitiendo, algunas personas notarán efectos beneficiosos y potenciadores de las experiencias sexuales, mientras que para otras la acción de la ketamina continuará siendo un impedimento para este tipo de actividades (Kelly, 1999).

En cualquier caso, los efectos más llamativos son la disociación y las alucinaciones. El consumidor de dosis altas generalmente tendrá la tendencia a cerrar los ojos, puede verse inmerso entonces en escenas que más allá de presentarse como imágenes muy vivas, darán la impresión de ser un marco real poblado muchas veces de entidades y seres con los que puede interactuar. Sería algo parecido a la realidad virtual, sólo que la experiencia puede parecer tan real que algunos usuarios creen a ciencia cierta haber visitado mundos paralelos y hablado con seres de otros planetas o de otras dimensiones. Así, algunos consumidores, más que como un agente psiquedélico, ven la ketamina como un instrumento o una llave que permite acceder a otras realidades tan tangibles y verdaderas como nuestro mundo de todos los días (Krupitsky \& Grinenko, 1997). Este tipo de creencias y alucinaciones se ven además potenciadas por los efectos disociativos de la sustancia. A dosis altas, la desconexión cuerpo-mente puede ser tal que el consumidor tenga la sensación de haber abandonado el propio cuerpo y llegue incluso a verlo mientras su mente flota en el espacio (Jansen, 2001). Esto puede facilitar el hecho de que las vívidas alucinaciones con las que uno mismo puede interactuar sean entendidas como visitas a mundos alternativos poblados de seres extraterrestres o entidades de dimensiones espirituales. Las experiencias de desdoblamiento corporal (OBEs: Out of Body Experiences) también pueden dar lugar a profundas experiencias místicas y a intensos viajes astrales, lo cual hace que la ketamina sea a veces una sustancia tentadora para personas interesadas en experimentar este tipo de vivencias (Moore \& Alltounian, 1978).

A nivel anecdótico, pero al mismo tiempo como confirmación de la ocurrencia de estos fenómenos, creo oportuno mencionar que los investigadores rusos Krupitsky y Grinenko (1997) que durante años realizaron un uso terapéutico de la ketamina en el tratamiento de pacientes alcohólicos, relataban que muchas de las personas que recibían inyecciones de ketamina para tratar su adicción, después de la administración decían entender el concepto cristiano de separación del alma y el cuerpo, conviene señalar en este punto que los pacientes eran rusos y generalmente ateos.

Otro fenómeno muy llamativo e impactante que puede darse con la administración de dosis altas de ketamina son las experiencias cercanas a la muerte (NDEs: Near Death Experiences). Este término, acuñado por Raymond A. Moody en su libro Vida Después de la Vida (1999), designa la experiencia de abandonar el propio cuerpo, creyendo firmemente que uno ha muerto, y viajar a través de un túnel hasta una luz brillante que generalmente se identifica con seres espirituales supremos.

Está ampliamente documentado (Jansen, 1996; 1997) que la administración de ketamina puede reproducir todos los fenómenos característicos de una NDE, y algunos investigadores (Jansen, 1996; 1997) han desarrollado teorías, apoyadas en datos científicos contrastados, que tratan de ofrecer una explicación neuroquímica de este fenómeno. 
Es interesante comentar que, en algunas personas, las experiencias cercanas a la muerte pueden tener un efecto terapéutico, en el sentido de que tras estas vivencias, llegan a reformular sus vidas de una forma muy positiva y optimista, viendo aumentada su alegría de vivir, reducido su miedo a la muerte y solucionados muchos de sus problemas personales e interpersonales (Jansen, 2001). No obstante, las OBEs y las NDEs también pueden ser auténticamente traumáticas y no todos los consumidores de ketamina viven la separación de su cuerpo o la convicción de que se han muerto como algo agradable o curioso, sino que para muchos estas experiencias son extremadamente angustiantes y terroríficas (Jansen, 2001).

\section{Remisión de los efectos y efectos secundarios}

Independientemente de cual haya sido la vía de administración, la remisión de los efectos no suele presentar grandes complicaciones, simplemente se van recuperando gradualmente los sentidos y quien haya consumido dosis altas irá recuperando la noción de lo que ha ocurrido, donde está, etc (Kelly, 1999). Gran parte de la experiencia psiquedélica permanecerá inaccesible a la memoria, es decir, se produciría una amnesia parcial que sólo permitiría recordar algunos aspectos o tramos de la misma (Jansen, 2001). Una vez terminada la experiencia psiquedélica, es frecuente que el usuario sienta mareos, vértigos, confusión, dolores de cabeza o nauseas, sobre todo si se mueve (Jansen, 2001). Por lo tanto, es conveniente que permanezca tranquilo una o dos horas más hasta que pase todo el efecto. Aún así, los efectos residuales no desaparecen del todo hasta las 24-48 horas (sobre todo con dosis altas), por lo que aún cuando el usuario pueda no verse del todo incapacitado al día siguiente, si es probable que se encuentre un poco aturdido.

\section{Riesgos del consumo}

Aún cuando el uso recreacional del clorhidrato de ketamina todavía no cuenta con una difusión masiva y aún se carece de estudios epidemiológicos, resulta posible mencionar determinados riesgos y complicaciones derivados del consumo, en cuanto se trata de una sustancia que ha sido utilizada en clínica durante más de treinta años. En este sentido, cabe destacar que su utilización está contraindicada en personas con hipertensión, problemas cardiacos o con antecedentes de accidentes cerebrovasculares, así como en pacientes sometidos a tratamiento con medicamentos tiroideos (Collins, 1996). Tampoco debe ser utilizada por personas con alteraciones psiquiátricas o con antecedentes de haberlas tenido (Collins, 1996). Su consumo durante el embarazo se desaconseja, en cuanto se sabe que atraviesa la placenta y alcanza en el feto una concentración similar a la de la madre, por lo que podría producir depresión fetal y aumento del tono uterino (Yate, 1996). No obstante, en ocasiones ha sido utilizada con éxito en obstetricia (Yate, 1996).

Por otra parte, aunque el margen de seguridad de la ketamina parece ser bastante amplio, puede producirse depresión respiratoria profunda sobre todo con la administración de dosis altas (Collins, 1996) o con el consumo concomitante de sustancias depresoras como alcohol, opiáceos o sedantes (Jansen, 2001). Del mismo modo, existe la posibilidad de que se produzcan convulsiones, derrames cerebrales, asfixia por espasmo laríngeo y paro cardiaco con resultado de muerte (Collins, 2001; Stockley, 1997). Físicamente también se pueden dar náuseas, vómitos, vértigo, lagrimeo, sudación, exceso de saliva, temblores, dolores abdominales incapacitantes y visión borrosa (Jansen, 2001). En algunos casos se han descrito aumentos bruscos de temperatura, lo cual podría dar lugar a una hipertermia maligna, especialmente si se mezcla con éxtasis y se realiza una actividad física continua como puede ser bailar (Jansen, 2001). En el plano físico, uno de los riesgos más graves asociados al uso recreacional deriva de la incapacidad física que provoca en el usuario (perdida de coordinación, dificultad para andar y moverse, etc.), dejándole sin recursos para reaccionar ante posibles accidentes y heridas, lo cual además se ve agravado por las propiedades analgésicas de la sustancia, que pueden dar lugar a que personas gravemente dañadas no sean conscientes de ello y empeoren su situación por no tomar rápidamente las medidas oportunas. En el plano psicológico, existe la posibilidad de que se desencadenen brotes psicóticos, ataques de pánico, crisis agudas de angustia y depresión, flashbacks y alteraciones del sueño (Jansen, 2001). El consumo crónico se ha asociado con problemas de memoria, dificultades de concentración, aprendizaje de nuevas situaciones, comprensión de metáforas y evaluación del propio comportamiento (White, 1998). Por último, la ketamina, además de producir una gran tolerancia con el consumo continuado, parece tener un alto potencial adictivo a nivel psicológico, calculándose que un 10-15\% de los consumidores Ilegan a cumplir los criterios diagnósticos de la dependencia (Jansen, 2001), incorporando su consumo a la rutina diaria y comportándose en todos los aspectos como lo pueda hacer un adicto a la heroína o a la cocaína. De hecho, con esta última sustancia comparte la característica de producir un intenso craving en los usuarios habituales, llevándoles en numerosas ocasiones a practicar auténticos atracones (binges) de ketamina, que a diferencia de los que se dan con la cocaína, no parecen producir el estado depresivo o apático posterior (crash). Esto parece ser debido a que cierto metabolito de la ketamina (la norketamina), tarda varios días en ser eliminado del organismo al tiempo que produce sus propios efectos, que serían concretamente irritabilidad y nerviosismo (Jansen, 2001). El hecho de que 
este metabolito tarde en ser eliminado, parece determinar también que no se produzca un síndrome de abstinencia físico como pueda ser el característico de los opiáceos (Jansen, 2001).

\section{CONCLUSIÓNES}

- La ketamina es un anestésico de confirmada utilidad en la práctica médica y veterinaria. No obstante, también están demostradas su capacidad para producir estados alterados de conciencia, sus marcadas propiedades psiquedélicas y su elevado potencial de abuso extrahospitalario.

- El uso psiconáutico en busca de experiencias trascendentales y de crecimiento personal se instauró de forma paralela a su implantación en la clínica durante los años 70. Sin embargo, este tipo de consumo se mantuvo circunscrito a reducidos círculos de usuarios iniciados en la cultura psiquedélica.

- Desde finales de los años 80, la ketamina comenzó a introducirse y extenderse lentamente en los entornos de ocio nocturno (especialmente raves), siendo objeto de un uso meramente recreativo.

- Los nombres que recibe el clorhidrato de ketamina en el argot callejero son K, Keta, Special-K, Super-K o Kit-Kat.

- Su presencia ha sido detectada en todos los continentes del planeta y en la mayoría de los países continúa siendo un medicamento de curso legal, es decir, no considerado como droga desde el punto de vista jurídico.

- Los efectos buscados por el consumidor recreacional son, fundamentalmente, la intensa y rápida embriaguez, las ligeras distorsiones perceptivas, la disociación, las alucinaciones y cierta euforia, así como contrarrestar los efectos secundarios desagradables derivados del uso de otras sustancias.

- En el ámbito recreacional generalmente se consumen dosis pequeñas o medianas (aunque muchas veces de forma repetida) y principalmente se utiliza la vía intranasal (esnifada) o la oral (bebida). No obstante, también se ha detectado el uso intramuscular entre círculos reducidos de usuarios recreativos.

- Los principales riesgos asociados a su uso y abuso son el desarrollo de una rápida tolerancia, el alto potencial de adicción psicológica, la facilidad para desencadenar heridas y accidentes cuando es consumida en un entorno inadecuado y la posibilidad de dar lugar a complicaciones psicológicas como crisis de ansiedad, depresión, estados psicóticos y flashbacks. También han de destacarse determinadas incompatibilidades médicas como la hiperten- sión, la presencia de alteraciones psiquiátricas o el tratamiento con medicamentos tiroideos.

- El consumo crónico se ha asociado, entre otras cosas, con problemas de memoria y concentración. Sería interesante, en este sentido, profundizar en las investigaciones sobre el potencial neurotóxico de la ketamina, así como de los aditivos y conservantes que habitualmente la acompañan en los preparados farmacéuticos.

- Dada la lenta pero continuada instauración y expansión del uso recreacional de la ketamina, sería deseable que desde el ámbito profesional se actualizasen los conocimientos al respecto y se siguiese de cerca la evolución de este fenómeno, a fin de poder actuar al mismo tiempo que se extendiese el consumo y no una vez que ya estuviese completamente instaurado y se hubiese convertido en un problema, lo cual, aún cuando no sabemos si realmente llegará a suceder, es evidentemente una posibilidad.

- En este sentido, son especialmente útiles los grupos de trabajo que intervienen a pie de campo, en los propios espacios de consumo, pues pueden detectar rápidamente los nuevos patrones de uso y realizar en tiempo real una actuación preventiva y de reducción de riesgos eficaz.

- Una de las labores fundamentales de estos grupos sería precisamente asesorar a aquellas personas que ya han decidido tomar la sustancia, sobre como llevar a la práctica unas pautas de consumo en las que los riesgos se vieran minimizados: principalmente evitando la mezcla con grandes cantidades de alcohol y adoptando un uso de pequeñas dosis únicas (una o dos administraciones) en lugar de varias dosis repetidas cada poco tiempo, como sucede con sustancias como la cocaína, pues de esta manera incrementan mucho los riesgos de desarrollar tolerancia y dependencia.

- En círculos reducidos, la utilización de la vía intramuscular también es un riesgo importante, pues se trata de una población escasamente informada y poco receptiva a las campañas dirigidas a la población heroinómana que tradicionalmente ha utilizado la vía endovenosa. Esto determina que, a veces, se den prácticas de riesgo como compartir las jeringuillas o no utilizar material estéril. Los grupos de reducción de riesgos pueden cumplir, en este sentido, una importante labor, en cuanto son los únicos que realmente pueden establecer contacto directo con estos consumidores. En consecuencia, en contextos específicos y con grupos muy concretos, se plantea la posibilidad de realizar una campaña informativa al respecto y, en última instancia, incluso dispensar jeringuillas gratuitamente, de forma controlada, seleccionada y discreta. 
- Por último, sería deseable incluir la ketamina en los estudios epidemiológicos sobre consumo de sustancias psicoactivas o bien realizar valoraciones en entornos específicos sobre la difusión del consumo recreativo y sobre los patrones de uso más extendidos.

\section{BIBLIOGRAFÍA}

BARAYUGA, D. 1997. State drug officials on the lookout for ketamina. Honolulu Star-Bulletin. http://starbulletin. com/97/04/21/news/story2.htm

BOOTH, N. H. 1988. Anestésicos intravenosos y otros anestésicos parenterales. En BOOTH, N. H. \& McDONALD, C. E. Eds.: Farmacología y Terapéutica Veterinaria, V 1. Acribia, S. A. Zaragoza. España. p 231-289.

CLOUD, J. 1997. "Is Your Kid On K?" Time Magazine, 20/10/1997, p 56-57.

COLLINS, V. 1996. Anestesia intravenosa: no barbitúricos-no narcóticos. En COLLINS, V. Eds,: Anestesiología. Anestesia General y Regional. McGraw-Hill-Interamericana. México. p 743-796

DALGARNO, P. J. \& SHEWAN, D. 1996. Ilícit use of ketamine in Scotland. Journal of Psychoactive Drugs 28, $\mathrm{p}$ 191-199.

DEA. 1999. Schedules of Controlled Substances: Placement of Ketamine into Schedule III. http://www.usdoj.gov/ dea/programs/diverson/ketamine.htm

DEAN, M. \& MORGENTHALER, J. 1998. GHB: The natural mood enhancer. Smart Publications. Petaluma, CA. 192 pags.

DIARIO 16. 1999. La ketamina, una droga peligrosa disponible sin control. Diario 16, 26-11.1999.

DIARIO 16. 1999. Las farmacias siguen dispensando ketamina pese a las medidas del Colegio profesional. Diario $16,27 / 11 / 1999$.

DIARIO 16. 2000. La OCU reitera que la ketamina se vende sin control en las farmacias. Diario 16, 21/01/2000.

DIARIO 16. 2000. EE.UU. alerta del tráfico de ketamina y España sigue sin controlar su venta. Diario 16, 26/01/2000.

DOMINO, E. F.; CHODOFF, P. \& CORSSEN, G. 1965. Pharmacological effects of $\mathrm{Cl}-581$, a new dissociative anesthetic, in man. Clinical and Pharmacological Therapeutics 6, p 279-261.

EL MUNDO. 1998. La comunidad avisa del peligro de dos drogas de moda: special k y hard rock. El Mundo. Domingo, 7 de junio de 1998.

EL PAÍS 1998. Cuernos de Espuma. El Páis de las Tentaciones 220, 9 de enero de 1998, portada y p 4.

EL PAÍS. 2001. Aumenta el uso de la ketamina como droga. El País, 08/05/2001.

EL PAÍS. 2001. Intervenido en Barcelona un gran alijo de ketamina, un anestésico veterinario que se utiliza como droga. El País, 21/11/2001.
ESCOHOTADO, A. 1998. Historia general de las drogas. Espasa Calpe, S. A. Madrid. 1542 pags.

ESTEBAN, J. 1998. Perspectivas futuras sobre drogas de diseño. Farmacéuticos 215, marzo. p 18-23

FERICGLA, J. M. 2000. El arduo problema de la terminología. Cáñamo. Especial 2000. La Cañamería Global. Barcelona. p 104-113.

GALLEGO, J. T. 2001. Ketamina y experiencias cercanas a la muerte. Cáñamo. Junio 2001, n 42. La Cañamería Global. Barcelona. p 98-104.

GAMELLA, J. F., ALVAREZ ROLDÁN, A. 1997. Drogas de Síntesis en España. Patrones y tendencias de adquisición y consumo. Plan Nacional Sobre Drogas. 386 pags.

GAYO, A. 1999. La droga de la Generación-K. Interviú 1.212, julio 1999, p 10-15.

HANSEN, G.; JENSEN, S.; CHANDRESH, L.; HILDEN, T. 1988. The psychotropic effect of ketamine. Journal of Psychoactive Drugs 20, p 419-425.

HIDALGO, E. 2000. Uso Recreacional del Clorhidrato de Ketamina. Boletín del Instituto para el Estudio de las Adicciones, julio del año 2000. http://w3.arrakis.es /iea/boletin/opinion.htm

HIDALGO, E. 2001. Ketamina: Usos y Abusos. Ulises: Revista de Viajes Interiores, 4, Primavera 2001. Los Libros de la Liebre de Marzo. Barcelona. p 24-31.

IPRC. 1997. New York passes tough ketamine law. Substance Abuse News Summary Service, 29/09/1997.

JAMES, J. 1999. Disco Bloodbath. Hodder and Stouughton. London. 238 pags.

JANSEN, K. L. R. 1996. Neuroscience, ketamine and the near-death experience: the role of glutamate and the NMDA receptor. En: L. J. Bailey \& J. Yates (Eds.) The Near-Death Experience: A Reader. New York: Routledge, p 264-282.

JANSEN., K. L. R. 1997. The ketamine model of the neardeath experience: a central role for the NMDA receptor. En: Journal of Near-Death Studies 16, 1, 5-27.

JANSEN, K.L.R. 2001. K-Ketamine, Dreams and Realities. United States: The Multidisciplinary Association for Psychedelic Studies. 354 pags.

KELLY, K. 1999. The Little Book of Ketamine. Ronin Publishing. Berkeley, California. 96 pags.

KUPRITSKY, E. M. \& GRINENKO, A. Y. 1997. Ketamine Psychedelic

Therapy (KPT): a review of the results of ten years of research. Journal of Psychoactive Drugs 29 (2): p 165183.

LEARY, T. 1997. Design for Dying. Thorsons. London. 239 pags.

LILLY, J. 1997. The Scientist: A metaphysical autobiography. Ronin Publishing. Berkeley, California. 208 pags.

LORENZO, P. 1988. MDMA y otras feniletilaminas. Farmacología y toxicología general. En: BOBES, J. \& LORENZO, P. Eds: Éxtasis (MDMA): un abordaje comprehensivo. Masson, S. A.. Barcelona. p 15-39. 
LUNA, A. \& SÁNCHEZ, C. 1998. Análisis toxicológico de los comprimidos de MDMA en España. En: BOBES, J. \& LORENZO, P. Eds: Extasis (MDMA): un abordaje comprehensivo. Masson, S. A. Barcelona. p 73-88.

MALINOVSKY, J. M.; LEAPAGE, J. Y. ET AL. 1993. Is ketamine or its preservative responsible for neurotoxicity in the rabbit? Anesthesiology, 78, n 1, jan 1993. p 109115.

MEDIA AWARENESS PROJECT. 1999. Miami police make largest "special-k" bust. http://www.mapinc.org/drugnews/u99/n1002/a08.htm

MOODY, R. A. 1999. Vida Después de la Vida. Edaf. Madrid. 208 pags.

MOORE, M. \& ALLTOUNIAN, H. 1978. Journeys Into The Bright World. Massachusetts: Para Research. 184 pags.

PLOURDE, G.; BARIBEAU, J.; BONHOMME, V. 1997. Ketamine increases the amplitude of the $40-\mathrm{Hz}$ auditory steady-state response in humans. British Journal of Anaesthesia 78 (5): p 524-529.

RAPOSO, C. 1998. Estimulantes: una llamada de atención. Farmacéuticos 215, marzo. p 40-48.

ROLLO, S. \& SAMORINI, G. 1999. Ketamina: el factor K de la psiquedelia. Ulises: revista de viajes interiores, 2
Primavera de 1999. Los Libros de la Liebre de Marzo. Barcelona. p 46-53

RUSHKOFF, D. 1997. The Ecstasy Club. Hodder and Stoughton. London. 315 pags.

SAMPER, J. 2001. Empleo Psiconáutico de la ketamina. Cañamo. Especial 2001. La Cañamería Global. Barcelona. p 214-218.

SPUTZ, R. 1989. I never met a reality I didn't like: A report on "Vitamin-K". High Times, october 1989, p 64-82.

STOCKLEY, D. 1997. Drogas: Guía Ilustrada para Agentes de Policía. Edex Kolektiboa. Bilbao. 189 pags.

TURNER, D. M. 1994. The Essential Psychedelic Guide. Panther Press, San Francisco, CA. http://squeaker.cat.net/ donut/guide/cover.html

TWEED, W. A.; MINICK, M. \& MYMIN, D. 1972. Circulatory responses to ketamine. Anesthesiology 37, p 613-619.

WEIL, A. \& ROSEN, W. 1999. Del café a la Morfina. RBA Libros S.A. Barcelona. 270 pags.

WHITE, W. 1998. This Is Your Brain On Dissociatives: The bad news is finally in. http://lycaeum.org/drugs/cyclohexamines/dissociative.brain.damage.html

YATE, P. 1996. Intravenous Anaesthesia. En KAUFMAN, L, Eds, : Pharmacology in the Practice of Anaesthesia. Arnold,. London. p 60-73. 
